Article

\title{
Fourier Analysis with Generalized Integration
}

\author{
Juan H. Arredondo ${ }^{1,+}$, Manuel Bernal ${ }^{1,+}$ and María Guadalupe Morales ${ }^{2, *,+}$ \\ 1 Departamento de Matemáticas, Universidad Autónoma Metropolitana—Iztapalapa, Av. San Rafael Atlixco 186, \\ México City 09340, Mexico; iva@xanum.uam.mx (J.H.A.); mbg@xanum.uam.mx (M.B.) \\ 2 Department of Mathematics and Statistics, Faculty of Science, Masaryk University, Kotlářská 2, \\ 61137 Brno, Czech Republic \\ * Correspondence: maciasm@math.muni.cz \\ + All authors contributed equally to this work.
}

Received: 24 June 2020; Accepted: 17 July 2020; Published: 21 July 2020

\begin{abstract}
We generalize the classic Fourier transform operator $\mathcal{F}_{p}$ by using the Henstock-Kurzweil integral theory. It is shown that the operator equals the HK-Fourier transform on a dense subspace of $\mathcal{L}^{p}, 1<p \leq 2$. In particular, a theoretical scope of this representation is raised to approximate the Fourier transform of functions on the mentioned subspace numerically. Besides, we show the differentiability of the Fourier transform function $\mathcal{F}_{p}(f)$ under more general conditions than in Lebesgue's theory. Additionally, continuity of the Fourier Sine transform operator into the space of Henstock-Kurzweil integrable functions is proved, which is similar in spirit to the already known result for the Fourier Cosine transform operator. Because our results establish a representation of the Fourier transform with more properties than in Lebesgue's theory, these results might contribute to development of better algorithms of numerical integration, which are very important in applications.
\end{abstract}

Keywords: fourier transform; Henstock-Kurzweil integral; bounded variation function; $\mathcal{L}^{p}$ spaces

MSC: 35S30; 26A39; 41A35; 34A25; 26A45

\section{Introduction}

The Lebesgue integral has strong implications in Fourier Analysis. Integration theory had an important development in the last half-century. Thereby, with the introduction of new integration theories, the possibility to extend fundamental results arises, allowing for new and better numerical approaches. For example, the Henstock-Kurzweil integral contains Riemann, improper Riemann, and Lebesgue integrals with the values of the integrals coinciding [1]. Thus, in [2] it was proved that, for subsets of $p$-integrable functions, $1<p \leq 2$, the classical Fourier transform, $\mathcal{F}_{p}(f)(s)$, is equal a.e. to a Henstock-Kurzweil integral, which has a pointwise expression for any $s \neq 0$, see Remark 1 and Theorem 2. Moreover, this representation allows for analyzing more properties related to the Fourier transform, as continuity or asymptotic behavior. On the other hand, Fourier Analysis is related to Approximation theory. Important applications are based on integration theory, for example, the approximation of the Fourier transform has implications in digital image processing, economic estimates, acoustic phonetics, among others [3,4].

In [5], the Fourier transform is studied for functions in $B V_{0}(\mathbb{R})$ (the space of bounded variation functions on $\mathbb{R}$ vanishing at infinite) whose derivative lies in the Hardy space $H^{1}(\mathbb{R}) \subsetneq$ $\mathcal{L}^{1}(\mathbb{R})$, where $\mathcal{L}^{1}(\mathbb{R})$ is the space of Lebesgue integrable functions. This operator is known as the Fourier-Stieltjes transform. In [6-10] the Fourier-Stieltjes transform was analyzed obtaining asymptotic formulas and integrability for the Fourier Cosine and Sine transforms of such kind of functions.

The generalizations of some classic notions in mathematics have always been an important issue. Sometimes these abstractions lead to equivalent definitions when some restrictions are taken into 
account. This opens up the possibility to consider a strictly larger class of functions where some calculus remains valid. In particular, this happens for extensions of the Fourier transform operator. For example, in the case of the work of T. Carleman and the later one of L. Scharwtz regarding generalized Fourier transformations. Both definitions are equivalent for temperate distributions, although very different in nature [11].

The present paper is organized, as follows. In Section 2, some notations are introduced, and we recall some preliminaries concepts regarding the Henstock-Kurzweil integral and the classical Fourier transform operator. In Section 3, we present the Fourier transform operator on nonclassical spaces of functions in the context of the Henstock-Kurzweil integral. In Section 4, we prove that the Fourier transform $\mathcal{F} p(f)$ keeps continuity and asymptotic behavior on subsets of $\mathcal{L}^{p}(\mathbb{R}) \backslash \mathcal{L}^{1}(\mathbb{R})$. In particular, a possible way to obtain a numerical approximation of $\mathcal{F} p(f)$ is shown via the Henstock-Kurzweil integral. In Section 5, we follow the line from [11] by extending the Fourier transform on $\mathcal{L}^{1}(\mathbb{R})$ to a strictly larger class of functions. This is facilitated through the use of the Henstock-Kurzweil integration theory in the framework of Fourier Analysis. Thus, we extend a classical theorem in Lebesgue's theory [12] [Theorem 9.2]. Besides, we give some examples to illustrate the applicability of our results. Finally, in Section 6, it is proved that the Fourier Sine transform is a continuous operator from a subspace of $\mathcal{L}^{2}(\mathbb{R})$ into $H K(\mathbb{R})$, which is a similar result to that obtained for the Fourier Cosine transform [13].

\section{Preliminaries}

We follow the notation from [14] to introduce basic definitions of the Henstock-Kurzweil integral. Let $\overline{\mathbb{R}}=\mathbb{R} \cup\{ \pm \infty\}$ and $[a, b]$ be a non-degenerative interval in $\overline{\mathbb{R}}$, a partition $P$ of $[a, b]$ is a finite collection of non-overlapping intervals, such that

$$
[a, b]=I_{1} \cup I_{2} \cup \ldots \cup I_{n} .
$$

Specifically, the partition itself is the set of endpoints of each sub-interval $I_{i}$,

$$
a=x_{0} \leq x_{1} \leq x_{2} \leq \ldots \leq x_{n}=b,
$$

where

$$
I_{i}:=\left[x_{i-1}, x_{i}\right] \text { for } i=1, \ldots, n .
$$

Observe that $[a, b]$ can be unbounded.

Definition 1. A tagged partition of $[a, b]$

$$
\dot{P}=\left\{\left(I_{i}, t_{i}\right)\right\}_{i=1}^{n}
$$

is a finite set of ordered pairs $\left\{\left(I_{i}, t_{i}\right)\right\}_{i=1}^{n}$, where the collection of subintervals $\left\{I_{i}\right\}$ forms a partition of $[a, b]$ and the point $t_{i} \in I_{i}$ is called a tag of $I_{i}$.

Definition 2. A map $\delta:[a, b] \rightarrow(0, \infty)$ is called gauge function on $[a, b]$. Given a gauge function $\delta$ on $[a, b]$, it is said that a tagged partition $\dot{P}=\left\{\left(\left[x_{i-1}, x_{i}\right] ; t_{i}\right)\right\}_{i=1}^{n}$ of $[a, b]$ is $\delta$-fine according to the following cases: For $a \in \mathbb{R}$ and $b=\infty$ :

1. $a=x_{0}, b=x_{n}=t_{n}=\infty$.

2. $\left[x_{i-1}, x_{i}\right] \subset\left[t_{i}-\delta\left(t_{i}\right), t_{i}+\delta\left(t_{i}\right)\right]$, for all $i=1,2, \ldots, n-1$.

3. $\left[x_{n-1}, \infty\right] \subset\left[\frac{1}{\delta\left(t_{n}\right)}, \infty\right]$.

For $a=-\infty$ and $b \in \mathbb{R}:$

1. $a=x_{0}=t_{1}=-\infty, b=x_{n}$. 
2. $\left[x_{i-1}, x_{i}\right] \subset\left[t_{i}-\delta\left(t_{i}\right), t_{i}+\delta\left(t_{i}\right)\right]$, for all $i=2, \ldots, n$.

3. $\left[-\infty, x_{1}\right] \subset\left[-\infty,-\frac{1}{\delta\left(t_{1}\right)}\right]$.

For $a=-\infty$ and $b=\infty$ :

1. $a=x_{0}=t_{1}=-\infty, b=x_{n}=t_{n}=\infty$.

2. $\left[x_{i-1}, x_{i}\right] \subset\left[t_{i}-\delta\left(t_{i}\right), t_{i}+\delta\left(t_{i}\right)\right]$, for all $i=2, \ldots, n-1$.

3. $\left[x_{n-1}, \infty\right] \subset\left[\frac{1}{\delta\left(t_{n}\right)}, \infty\right]$ and $\left[-\infty, x_{1}\right] \subset\left[-\infty,-\frac{1}{\delta\left(t_{1}\right)}\right]$.

For $a, b \in \mathbb{R}:$

1. $\left[x_{i-1}, x_{i}\right] \subset\left[t_{i}-\delta\left(t_{i}\right), t_{i}+\delta\left(t_{i}\right)\right]$, for all $i=1,2, \ldots, n$.

According to the convention concerning to the "arithmetic" in $\overline{\mathbb{R}}, 0 \cdot( \pm \infty)=0$, a real-valued function $f$ defined over $\mathbb{R}$ can be extended by setting $f( \pm \infty)=0$. Thus, the definition of the Henstock-Kurzweil integral over intervals is introduced in $\overline{\mathbb{R}}$.

Definition 3. Let $[a, b]$ be an interval in $\overline{\mathbb{R}}$. The real-valued function $f$ defined over $[a, b]$ is said to be Henstock-Kurzweil integrable on $[a, b]$ iff there exists $A \in \mathbb{R}$, such that, for every $\epsilon>0$, there exists a gauge function $\delta_{\epsilon}$ over $[a, b]$, such that if $\dot{P}=\left\{\left(\left[x_{i-1}, x_{i}\right] ; t_{i}\right)\right\}_{i=1}^{n}$ is a $\delta_{\epsilon}$-fine partition of $[a, b]$, then

$$
\left|\sum_{i=1}^{n} f\left(t_{i}\right)\left(x_{i}-x_{i-1}\right)-A\right|<\epsilon .
$$

The number $A$ is the integral of $f$ over $[a, b]$ and it is denoted by $\int_{a}^{b} f(x) d x=A$.

The set of all Henstock-Kurzweil integrable functions on the interval $I$ is denoted by $H K(I)$, and the set of Henstock-Kurzweil integrable functions over each compact interval is denoted by $H K_{\text {loc }}(\mathbb{R})$. The integrals will be in the Henstock-Kurzweil sense, if not specified.

The following result is well known as the Hake Theorem and it is an useful characterization of the Henstock-Kurzweil integrable functions.

Theorem 1 ([14]). Let $I:=[a, b]$ and $f: I \rightarrow \mathbb{R}$. Subsequently, the following statements are equivalent:

1. $f \in H K(I)$ and $\int_{a}^{b} f(x) d x=A$.

2. $f \in H K[c, d]$ for every compact interval $[c, d] \subset[a, b]$ and

$$
\lim _{c \rightarrow a^{+}, d \rightarrow b^{-}} \int_{c}^{d} f(x) d x=A .
$$

The Multiplier Theorem in [14] states that the bounded variation functions are the multipliers of the Henstock-Kurzweil integrable functions. Moreover, this concept is related to the Riemann-Stieltjes integral, which generalizes the Riemann integral and, also, it is useful to calculate the Fourier transform, see Theorem 3 below. There exist several versions of the Riemann-Stieltjes integral. In this work, it is considered the Riemann-Stieljes $(\delta)$-integral, also called norm Riemann-Stieltjes integral $[15,16]$. Here, for simplicity, it is called as Riemann-Stieltjes integral.

\subsection{The Fourier Transform Operator in the Classical Sense}

We will enunciate basic results regarding the Fourier Analysis in the classical sense, i.e., considering Lebesgue's integral.

Definition 4. Let $1 \leq p<\infty$. For any Lebesgue measurable function $f: \mathbb{R} \rightarrow \mathbb{R}$, we define

$$
\|f\|_{p}=\left[\int_{\mathbb{R}}|f|^{p} d \mu\right]^{\frac{1}{p}}
$$


For each $p \geq 1$, the set of functions $f$, such that $\|f\|_{p}<\infty$ (called $p$-integrable functions) is a normed space (considering equivalence classes with respect to $\|\cdot\|_{p}$ ) and it is denoted by $\mathcal{L}^{p}(\mathbb{R})$.

The Fourier transform has been developed in the context of the Lebesgue theory and over the spaces $\mathcal{L}^{p}(\mathbb{R})$, with important implications in different areas, e.g., optics, signal theory, statistics, probability theory [3].

The set $\mathcal{L}^{1}(\mathbb{R})$ is well known as the space of Lebesgue integrable functions on $\mathbb{R}$ or absolutely integrable functions. $\mathcal{L}^{2}(\mathbb{R})$ is a normed space with an inner product that provides algebraic and geometric techniques that are applicable to spaces of arbitrary dimension. These spaces are usually considered to define the Fourier transform $[12,17,18]$.

Definition 5. Let $f \in \mathcal{L}^{1}(\mathbb{R})$. The Fourier transform of $f$ at the point $s$ is defined as

$$
\mathcal{F}_{1}(f)(s)=\frac{1}{\sqrt{2 \pi}} \int_{-\infty}^{\infty} e^{-i s x} f(x) d x .
$$

It is well known that $\mathcal{F}_{1}(f)$ is pointwise defined and by the Riemann-Lebesgue Lemma, $\mathcal{F}_{1}(f)$ belongs to $C_{\infty}(\mathbb{R})$, the set of complex-valued continuous functions on $\mathbb{R}$ vanishing at $\pm \infty[2,18]$. On the other hand, the space $\mathcal{L}^{2}(\mathbb{R})$ is not contained in $\mathcal{L}^{1}(\mathbb{R})$. Thus, the operator $\mathcal{F}_{1}$ is not well defined over $\mathcal{L}^{2}(\mathbb{R})$. Nevertheless, the Fourier transform on $\mathcal{L}^{2}(\mathbb{R})$ is given as an extension of the Fourier transform $\mathcal{F}_{1}(f)$ initially defined on $\mathcal{L}^{1}(\mathbb{R}) \cap \mathcal{L}^{2}(\mathbb{R})$. It means that the Fourier transform over $\mathcal{L}^{2}(\mathbb{R})$ is defined as a limit [19], and it is denoted as $\mathcal{F}_{2}$.

Definition $6([17,18,20])$. The Fourier transform operator in $\mathcal{L}^{p}(\mathbb{R}), 1<p<2$, is given as

$$
\begin{gathered}
\mathcal{F}_{p}: \mathcal{L}^{p}(\mathbb{R}) \rightarrow \mathcal{L}^{q}(\mathbb{R}), \\
\mathcal{F}_{p}(f)=\mathcal{F}_{1}\left(f_{1}\right)+\mathcal{F}_{2}\left(f_{2}\right),
\end{gathered}
$$

where $p^{-1}+q^{-1}=1, f_{1} \in \mathcal{L}^{1}(\mathbb{R}) \cap \mathcal{L}^{p}(\mathbb{R}), f_{2} \in \mathcal{L}^{2}(\mathbb{R}) \cap \mathcal{L}^{p}(\mathbb{R})$ and $f=f_{1}+f_{2}$.

\section{HK-Fourier Transform}

At the beginning of this century, the Fourier theory was developed using the Henstock-Kurzweil theory. In [21], E. Talvila showed some existence theorems and continuity of the Fourier transform over Henstock-Kurzweil integrable functions. Moreover, the Fourier transform has been studied as a Henstock-Kurzweil integral over non-classical spaces of functions [2,22]. It is well known that if $I$ is a compact interval, then

$$
B V(I) \subset \mathcal{L}^{1}(I) \subset H K(I),
$$

where $B V(I)$ denotes the set of bounded variation functions on $I$. However, when $I$ is an unbounded interval,

$$
B V(I) \nsubseteq \mathcal{L}^{1}(I)
$$

and

$$
\mathcal{L}^{1}(I) \nsubseteq \subseteq H K(I) \cap B V(I),
$$

the definition of bounded variation functions on unbounded intervals can be found in $[2,13,22]$. Thereby, when $I$ is unbounded, there is no inclusion relation between $\mathcal{L}^{1}(I)$ and $H K(I) \cap B V(I)$. On the other hand, $B V(I) \cap H K(I) \subset \mathcal{L}^{2}(I)$ (by the Multiplier Theorem [14]). In [23] [Lemma 4.1], it is proved that $B V(I) \cap H K(I) \subset B V_{0}(I)$, where $B V_{0}(I)$ denotes the subspace of $B V(I)$ consisting of the functions that have limit zero at $\pm \infty$. Accordingly, it is possible to analyze the Fourier transform via 
the Henstock-Kurzweil integral over $B V_{0}(\mathbb{R})$. In [22], it was shown a generalized Riemann-Lebesgue Lemma on unbounded intervals, giving rise to the definition of the HK-Fourier transform [2].

Definition 7. $\mathcal{L}^{1}(\mathbb{R})+B V_{0}(\mathbb{R})$ denotes the vector space of functions $f=f_{1}+f_{2}$, where $f_{1} \in \mathcal{L}^{1}(\mathbb{R})$ and $f_{2} \in B V_{0}(\mathbb{R})$.

Definition 8. The HK-Fourier transform is defined as

$$
\begin{aligned}
& \mathcal{F}_{H K}: \mathcal{L}^{1}(\mathbb{R})+B V_{0}(\mathbb{R}) \rightarrow C_{\infty}(\mathbb{R} \backslash\{0\}), \\
& \mathcal{F}_{H K}(f)(s):=\frac{1}{\sqrt{2 \pi}} \int_{\mathbb{R}} e^{-i s x} f(x) d x \\
&=\frac{1}{\sqrt{2 \pi}} \int_{\mathbb{R}} \cos (s x) f(x) d x-i \frac{1}{\sqrt{2 \pi}} \int_{\mathbb{R}} \sin (s x) f(x) d x \\
& \equiv \mathcal{F}_{H K}^{C}(f)(s)-i \mathcal{F}_{H K}^{S}(f)(s),
\end{aligned}
$$

where the integrals are in Henstock-Kurzweil sense. $\mathcal{F}_{H K}^{C}(f)$ and $\mathcal{F}_{H K}^{S}(f)$ are called the HK-Fourier Cosine and the HK-Fourier Sine transforms of $f$, respectively.

Proposition 1. The HK-Fourier transform is well defined.

Proof. Suppose that $f=u_{1}+v_{1}=u_{2}+v_{2}$ with $u_{i} \in \mathcal{L}^{1}(\mathbb{R})$ and $v_{i} \in B V_{0}(\mathbb{R})$ for $i=1,2$. Therefore,

$$
u_{1}-u_{2}=v_{2}-v_{1} \in \mathcal{L}^{1}(\mathbb{R}) \cap B V_{0}(\mathbb{R}) .
$$

This yields the result since the Henstock-Kurzweil integral coincides with the Lebesgue integral on the intersection $\mathcal{L}^{1}(\mathbb{R}) \cap B V_{0}(\mathbb{R})$, see [2]. Therefore, $\mathcal{F}_{H K}(f)$ does not depend on the representation of the function $f$.

Remark 1. Note that some integrals in (1) might not converge at $s=0$. By [22] [Theorem 2.5] the HK-Fourier Cosine and Sine transforms of any function $f$ in $B V_{0}(\mathbb{R}), \mathcal{F}_{H K}^{C}(f)$, and $\mathcal{F}_{H K}^{S}(f)$ are well-defined continuous functions (except at $s=0$ ) and vanish at infinity as $o\left(|s|^{-1}\right.$ ).

The following result was proven in [2] [Theorem 3.3, Corollary 1].

Theorem 2. If $f \in \mathcal{L}^{p}(\mathbb{R}) \cap\left(\mathcal{L}^{1}(\mathbb{R})+B V_{0}(\mathbb{R})\right)$, for $1<p \leq 2$, then

$$
\mathcal{F}_{H K}(f) \in \mathcal{L}^{q}(\mathbb{R}) \cap C_{\infty}(\mathbb{R} \backslash\{0\}),
$$

where $p^{-1}+q^{-1}=1$. Moreover,

$$
\mathcal{F}_{p}(f)(s)=\mathcal{F}_{H K}(f)(s),
$$

almost everywhere. In particular, if $f \in B V_{0}(\mathbb{R})$, then

$$
\mathcal{F}_{H K}(f) \in C_{\infty}(\mathbb{R} \backslash\{0\}) .
$$

\section{An Approach of $\mathcal{F}_{p}$ via $\mathcal{F}_{H K}$}

According to Lebesgue's theory of integration, it is not always possible to achieve a pointwise expression of the Fourier transform operator $\mathcal{F}_{p}$, for $1<p \leq 2$. Nevertheless, there exist functions that belong to $\mathcal{L}^{p}(\mathbb{R}) \cap B V_{0}(\mathbb{R}) \backslash \mathcal{L}^{1}(\mathbb{R})$. In accordance with Theorem 2, we can apply 
the Henstock-Kurzweil integral in order to approach the Fourier transform operator $\mathcal{F}_{p}$ on subsets of $\mathcal{L}^{p}(\mathbb{R}) \backslash \mathcal{L}^{1}(\mathbb{R})$.

The set of absolutely continuous functions over each compact interval is denoted by $A C_{\text {loc }}(\mathbb{R})[5,9,24-26]$.

Theorem 3. If $\phi \in \mathcal{L}^{p}(\mathbb{R}) \cap B V_{0}(\mathbb{R}) \cap A C_{\text {loc }}(\mathbb{R})$, for $1 \leq p \leq 2$, then

1. $\mathcal{F}_{H K}(\phi) \in C_{\infty}(\mathbb{R} \backslash\{0\})$.

2. $\mathcal{F}_{H K}(\phi)(s)=\mathcal{F}_{p}(\phi)(s)$ a.e.

3. For every $s \in \mathbb{R} \backslash\{0\}$,

4. Moreover,

$$
\mathcal{F}_{H K}(\phi)(s)=-\frac{i}{s} \mathcal{F}_{1}\left(\phi^{\prime}\right)(s)
$$

$$
\left|\mathcal{F}_{H K}(\phi)(s)\right| \leq \frac{1}{\sqrt{2 \pi}} \cdot \frac{1}{|s|}\left\|\phi^{\prime}\right\|_{1}
$$

Proof. Let $\phi \in \mathcal{L}^{2}(\mathbb{R}) \cap B V_{0}(\mathbb{R}) \cap A C_{\text {loc }}(\mathbb{R})$. By Theorem 2, we get claims 1. and 2. Note that $\mathcal{F}_{H K}(\phi)(s)$ is defined for any $s \neq 0$, whereas $\mathcal{F}_{p}(\phi)(s)$ is defined almost everywhere. Applying the Theorem 1, we get,

$$
\mathcal{F}_{H K}(\phi)(s)=\frac{1}{\sqrt{2 \pi}} \lim _{T \rightarrow \infty}\left[\int_{-T}^{T} \cos (s t) \phi(t) d t-i \int_{-T}^{T} \sin (s t) \phi(t) d t\right] .
$$

From the Multiplier Theorem (see Theorem 10.12 in [14] [Sec. 10, p. 161]) and the hypothesis for $\phi$, we have

$$
\int_{\mathbb{R}} \cos (s t) \phi(t) d t=-\lim _{T \rightarrow \infty} \int_{-T}^{T}\left[\frac{\sin (s t)+\sin (s T)}{s}\right] d \phi .
$$

Similarly, for the Fourier Sine transform we get

$$
\int_{\mathbb{R}} \sin (s t) \phi(t) d t=-\lim _{T \rightarrow \infty} \int_{-T}^{T}\left[\frac{-\cos (s t)+\cos (s T)}{s}\right] d \phi,
$$

where we used that $\phi \in B V_{0}(\mathbb{R})$ vanishes at infinity. This yields, from (3),

$$
\begin{aligned}
\mathcal{F}_{H K}(\phi)(s)= & -\lim _{T \rightarrow \infty} \frac{1}{\sqrt{2 \pi}} \int_{-T}^{T} \frac{\sin (s t)+\sin (s T)}{s} d \phi \\
& +i \lim _{T \rightarrow \infty} \frac{1}{\sqrt{2 \pi}} \int_{-T}^{T} \frac{-\cos (s t)+\cos (s T)}{s} d \phi .
\end{aligned}
$$

Note that the Stieltjes-type integrals above exist as Riemann-Stieltjes and Lebesgue-Stieltjes integrals $[15,27,28]$. Because $\phi \in A C_{\text {loc }}(\mathbb{R})$, by [15] [Theorem 6.2.12] and [16] [Exercise 2, p. 186], it follows that

$$
\int_{-T}^{T}(\sin (s t)+\sin (s T)) d \phi=\int_{-T}^{T}(\sin (s t)+\sin (s T)) \phi^{\prime}(t) d t
$$

and

$$
\int_{-T}^{T}(-\cos (s t)+\cos (s T)) d \phi=\int_{-T}^{T}(-\cos (s t)+\cos (s T)) \phi^{\prime}(t) d t
$$


Because $\phi \in B V_{0}(\mathbb{R}) \cap A C_{l o c}(\mathbb{R})$, one gets

$$
\lim _{T \rightarrow \infty}\left[(\cos (s T)-\sin (s T)) \int_{-T}^{T} \phi^{\prime}(t) d t\right]=0,
$$

and [25] [Corollary 2.23] implies that $\phi^{\prime} \in \mathcal{L}^{1}(\mathbb{R})$. Therefore, we get

$$
\begin{aligned}
\mathcal{F}_{H K}(\phi)(s) & =\frac{1}{\sqrt{2 \pi}} \frac{1}{s} \lim _{T \rightarrow \infty}\left[-\int_{-T}^{T}[\sin (s t)+i \cos (s t)] \phi^{\prime}(t) d t\right] \\
& =\frac{1}{i s} \mathcal{F}_{1}\left(\phi^{\prime}\right)(s) .
\end{aligned}
$$

Furthermore,

$$
\left|\mathcal{F}_{H K}(\phi)(s)\right| \leq \frac{1}{\sqrt{2 \pi}} \cdot \frac{1}{|s|}\left\|\phi^{\prime}\right\|_{1} .
$$

For $1 \leq p<2$, the same formulas and argumentation are valid.

Remark 2. Because $f \in B V_{0}(\mathbb{R}) \cap A C_{\text {loc }}(\mathbb{R})$, by [14] [Theorem 7.5, $p$. 281] and [25] [Theorem 3.39], we have that $\left\|\phi^{\prime}\right\|_{1}=\operatorname{Var}(\phi, \mathbb{R})$ and $\phi \in A C(\mathbb{R})$. Thus, $B V_{0}(\mathbb{R}) \cap A C(\mathbb{R})=B V_{0}(\mathbb{R}) \cap A C_{\text {loc }}(\mathbb{R})$.

From Theorem 3, we have the following result.

Corollary 1. Let $\phi \in \mathcal{L}^{p}(\mathbb{R}) \cap B V_{0}(\mathbb{R}) \cap A C_{\text {loc }}(\mathbb{R})$, for $1 \leq p \leq 2$.

1. If $\phi$ is an even function, then

$$
\mathcal{F}_{H K}(\phi)(s)=-\sqrt{\frac{2}{\pi}} \cdot \frac{1}{s} \int_{0}^{\infty} \sin (s t) \phi^{\prime}(t) d t .
$$

2. If $\phi$ is an odd function, then

$$
\mathcal{F}_{H K}(\phi)(s)=-i \sqrt{\frac{2}{\pi}} \cdot \frac{1}{s} \int_{0}^{\infty}[\cos (s t)-1] \phi^{\prime}(t) d t .
$$

In either case, $\mathcal{F}_{H K}(\phi)(s)=\mathcal{F}_{p}(\phi)(s)$ a.e., where $\mathcal{F}_{H K}(\phi) \in C_{\infty}(\mathbb{R} \backslash\{0\})$.

E. Liflyand in [7-9] worked on a subspace of $B V_{0}(\mathbb{R}) \cap A C_{l o c}(\mathbb{R})$ to obtain integrability and asymptotic formulas for the Fourier transform. We restrict the domain of the Fourier transform operator to provide new integral expressions of the Fourier transform $\mathcal{F}_{p}$.

The implications from these results are that the classical Fourier transform $\mathcal{F}_{p}(f)(s)$ for $f$ in a dense subspace of $\mathcal{L}^{p}(\mathbb{R})$ is represented by a Lebesgue integral, is a continuous function, except at $s=0$, and it vanishes at infinity as $o\left(|s|^{-1}\right)$.

The algorithms of numerical integration are very important in applications, for example, the approximation of the Fourier transform has implications in digital image processing, economic estimates, acoustic phonetics, among others [3,4]. There exist integrable functions whose primitives cannot be explicitly calculated; thus, numerical integration is fundamental for achieving explicit results.

Additionally, note that the Lebesgue integral is not suitable for numerical approximations. On the other hand, (2), (5) and (6) provide expressions that might be used to approximate numerically $\mathcal{F}_{p}(f)$ at specific values. Actually, as a consequence of the Hake Theorem, it is possible to approximate $\mathcal{F}_{H K}(\phi)(s)$ via the relation 


$$
\mathcal{F}_{H K}(\phi)(s) \approx \frac{1}{\sqrt{2 \pi}} \int_{|t| \leq M} e^{-i s t} \phi(t) d t \quad(M \rightarrow \infty),
$$

for any $s \neq 0, \phi \in \mathcal{L}^{p}(\mathbb{R}) \cap B V_{0}(\mathbb{R}) \backslash \mathcal{L}^{1}(\mathbb{R})(1<p \leq 2)$. Moreover, Theorem 3 justifies and assures that $\mathcal{F}_{p}(\phi)(s)$ is asymptotically approximated by $(7)$. Note that Lebesgue's theory of integration only assures convergence of the integrals in (7) for a sequence of values of $M$ and $s$ in some (unknown) subset $\mathfrak{A} \varsubsetneqq \mathbb{R}$.

\section{Differentiability of the Fourier Transform}

A classical theorem in Lebesgue's theory is about differentiability under the integral sign [12,29]. The following result is a generalization of this, first we introduce the concept of a generalized absolutely continuous function in the restricted sense.

Definition 9. Let $I \subset \mathbb{R}$ be a compact interval. A function $\phi: I \rightarrow \mathbb{R}$ is absolutely continuous in the restricted sense on I if, for each $\epsilon>0$, there exists $\delta>0$ such that

$$
\sum_{i=1}^{l} w\left(\phi,\left[c_{i}, d_{i}\right]\right)<\delta
$$

whenever $\left\{\left[c_{i}, d_{i}\right]\right\}_{i=1}^{l}$ is a finite collection of non-overlapping intervals that have end points in I and satisfy

$$
\sum_{i=1}^{l}\left(d_{i}-c_{i}\right)<\delta
$$

where

$$
w(\phi,[a, b]):=\sup \{|\phi(y)-\phi(x)|, a \leq x \leq y \leq b\} .
$$

The space of all absolutely continuous functions in the restricted sense on $I$ is denoted by $A C^{*}(I) . A$ function $\phi: I \rightarrow \mathbb{R}$ is generalized absolutely continuous in the restricted sense on I if $\phi$ is continuous on $I$ and I can be written as a countable union of sets on each of which $\phi$ is $A C^{*}$. The space of all generalized absolutely continuous functions in the restricted sense on $I$ is denoted by $A C G^{*}(I)$.

A characterization of the Henstock-Kurzweil integrable functions is given by the generalized absolutely continuous functions in the restricted sense, due to the primitives of Henstock-Kurzweil integrable functions are $A C G^{*}$ functions, [24]. Thus, the primitives of locally Henstock-Kurzweil integrable functions are locally generalized absolutely continuous functions in the restricted sense on $\mathbb{R}$, and this space is denoted by $A C G_{l o c}^{*}(\mathbb{R})$.

Theorem 4. Let $f \in \mathcal{L}^{1}(\mathbb{R})+B V_{0}(\mathbb{R})$ such that $g(t):=t f(t)$ belongs to $\mathcal{L}^{1}(\mathbb{R})+B V_{0}(\mathbb{R})$. Subsequently, $\mathcal{F}_{H K}(f)$ is continuously differentiable away from zero and

$$
\frac{d}{d s} \mathcal{F}_{H K}(f)(s)=-i \mathcal{F}_{H K}(g)(s), \quad(s \neq 0) .
$$

Proof. For the case

$$
f, g \in B V_{0}(\mathbb{R}),
$$

let us define $G(s, t):=\cos (s t) f(t)$ in $\mathcal{L}_{\text {loc }}^{1}(\mathbb{R})$ with respect to $s$ for all $t \in \mathbb{R}$. Let $[\alpha, \beta]$ be any compact interval such that $0 \notin[\alpha, \beta]$ and let us consider the sequence $\left(\Phi_{n}\right)$ where

$$
\Phi_{n}(s):=\int_{-n}^{n} \frac{d}{d s} G(s, t) d t
$$


with $n \in \mathbb{N}$. Since

$$
\left|\Phi_{n}(s)\right| \leq \frac{2}{|s|} \operatorname{Var}(g, \mathbb{R}),
$$

then $\Phi_{n} \subset L^{1}[\alpha, \beta]$. Applying the Dominated Convergence Theorem, Fubini's Theorem, and Hake's Theorem [14], we get

$$
\begin{aligned}
\frac{1}{\sqrt{2 \pi}} \int_{\alpha}^{\beta} \int_{-\infty}^{\infty} \frac{d}{d s} G(s, t) d t d s & =\frac{1}{\sqrt{2 \pi}} \int_{\alpha}^{\beta} \int_{-\infty}^{\infty}-\sin (s t) g(t) d t d s \\
& =\frac{1}{\sqrt{2 \pi}} \int_{\alpha}^{\beta} \lim _{n \rightarrow \infty} \Phi_{n}(s) d s \\
& =\frac{1}{\sqrt{2 \pi}} \lim _{n \rightarrow \infty} \int_{-n}^{n} f(t)[\cos (\beta t)-\cos (\alpha t)] d t \\
& =\mathcal{F}_{H K}^{C}(f)(\beta)-\mathcal{F}_{H K}^{C}(f)(\alpha) .
\end{aligned}
$$

On the other hand,

$$
\begin{aligned}
\frac{1}{\sqrt{2 \pi}} \int_{-\infty}^{\infty} \int_{\alpha}^{\beta} \frac{d}{d s} G(s, t) d s d t & =\frac{1}{\sqrt{2 \pi}} \int_{-\infty}^{\infty} \cos (\alpha t) f(t)-\cos (\beta t) f(t) d t \\
& =\mathcal{F}_{H K}^{C}(f)(\beta)-\mathcal{F}_{H K}^{C}(f)(\alpha) .
\end{aligned}
$$

From (10), (14), and [26] [Theorem 4], we get that the HK-Fourier Cosine transform is differentiable under the integral sign. Because $g \in B V_{0}(\mathbb{R})$, by Theorem $2, \mathcal{F}_{H K}^{C}(f)^{\prime}$ is a continuous function (except at $s=0$ ) vanishing at infinity. By similar arguments, $\mathcal{F}_{H K}^{S}(f)^{\prime}(s)=\mathcal{F}_{H K}^{C}(g)(s)$ for any $s \neq 0$.

For the general case, we suppose $t f=g_{1}+g_{2} \in \mathcal{L}^{1}(\mathbb{R})+B V_{0}(\mathbb{R})$. Subsequently, (9) with $g=g_{1}+g_{2}$ also obeys $\left\{\Phi_{n}\right\} \subset L^{1}[\alpha, \beta]$, so that (10) remains valid. Therefore, the HK-Fourier transform is differentiable and (8) is obtained.

Corollary 2. Under the assumptions of Theorem 4. Subsequently,

$$
\mathcal{F}_{H K}^{S}(f) \in A C G_{l o c}^{*}(\mathbb{R}) .
$$

Proof. Theorem 4 implies that $\mathcal{F}_{H K}^{S}(f)$ is a continuously differentiable function away from zero, and [13] [Corollary 1] yields that its derivate is actually a function in $H K_{\text {loc }}(\mathbb{R})$. Therefore, [26] [Theorem 2] gives the result.

We will extend this theorem to study the differentiability of the Fourier transform $\mathcal{F}_{p}(f)$ for $1 \leq p \leq 2$. First, we present auxiliary results.

Theorem 5 ([12]). If $1 \leq p \leq \infty$ and $\left\{f_{n}\right\}$ is a Cauchy sequence in $\mathcal{L}^{p}(\mathbb{R})$, with limit $f$, then $\left\{f_{n}\right\}$ has a subsequence which converges to $f$ pointwise almost everywhere.

Lemma 1. Suppose that $1 \leq p \leq 2$ and $f \in \mathcal{L}^{p}(\mathbb{R})$. Afterwards, there exists a subsequence $\left(n_{k}\right) \in \mathbb{N}$, such that

$$
\frac{1}{\sqrt{2 \pi}} \lim _{k \rightarrow \infty} \int_{-n_{k}}^{n_{k}} e^{-i s x} f(x) d x=\mathcal{F}_{p}(f)(s)
$$

almost everywhere on $\mathbb{R}$.

Proof. The cases $p=1$ or $p=2$ follow from [12,18]. For $1<p<2$, due to $[2,17,20]$ there exist functions $f_{1} \in \mathcal{L}^{1}(\mathbb{R}) \cap \mathcal{L}^{p}(\mathbb{R}), f_{2} \in \mathcal{L}^{2}(\mathbb{R}) \cap \mathcal{L}^{p}(\mathbb{R})$, such that $f=f_{1}+f_{2}$. It follows that 


$$
\mathcal{F}_{p}(f)=\mathcal{F}_{1}\left(f_{1}\right)+\mathcal{F}_{2}\left(f_{2}\right)
$$

Applying once again $[12,18]$, we obtain a sequence $\left(n_{k}\right) \subset \mathbb{N}$, such that

$$
\frac{1}{\sqrt{2 \pi}} \lim _{k \rightarrow \infty} \int_{-n_{k}}^{n_{k}} e^{-i s x} f(x) d x=\mathcal{F}_{2}\left(f_{2}\right)(s),
$$

almost everywhere on $\mathbb{R}$. This yields,

$$
\begin{aligned}
\frac{1}{\sqrt{2 \pi}} \lim _{k \rightarrow \infty} \int_{-n_{k}}^{n_{k}} e^{-i s x} f(x) d x & =\frac{1}{\sqrt{2 \pi}} \lim _{k \rightarrow \infty} \int_{-n_{k}}^{n_{k}} e^{-i s x} f_{1}(x) d x \\
& +\frac{1}{\sqrt{2 \pi}} \lim _{k \rightarrow \infty} \int_{-n_{k}}^{n_{k}} e^{-i s x} f_{2}(x) d x \\
& =\mathcal{F}_{1}\left(f_{1}\right)(s)+\mathcal{F}_{2}\left(f_{2}\right)(s) \\
& =\mathcal{F}_{p}(f)(s),
\end{aligned}
$$

almost everywhere. This proves the statement.

Below we use the notation $p^{-1}+q^{-1}=1$.

Proposition 2. Let $1 \leq p \leq 2$ be fixed. If $f \in \mathcal{L}^{p}(\mathbb{R})$ and $g(t):=t f(t)$ belongs to $\mathcal{L}^{1}(\mathbb{R})+B V_{0}(\mathbb{R})$, then by redefining $\mathcal{F}_{p}(f)(s)$ on a set of measure zero, it yields

$$
\frac{d}{d s} \mathcal{F}_{p}(f)(s)=-i \mathcal{F}_{H K}(g)(s), \quad(s \neq 0) .
$$

Proof. Take values $s=\alpha$, and $s=\beta$ such that (12) is valid. Suppose that $0<\alpha<\beta$. Proceeding similarly as in Theorem 4 , we have

$$
\begin{aligned}
-i \int_{\alpha}^{\beta} \mathcal{F}_{H K}(g)(s) d s & =\int_{\alpha}^{\beta} \lim _{k \rightarrow \infty} \frac{-i}{\sqrt{2 \pi}} \int_{-n_{k}}^{n_{k}} e^{-i s t} t f(t) d t d s \\
& =\frac{-i}{\sqrt{2 \pi}} \lim _{k \rightarrow \infty} \int_{-n_{k}}^{n_{k}} \int_{\alpha}^{\beta} e^{-i s t} t f(t) d s d t \\
& \left.=\frac{-i}{\sqrt{2 \pi}} \lim _{k \rightarrow \infty} \int_{-n_{k}}^{n_{k}}\left(e^{-i \beta t}-e^{-i \alpha t}\right) f(t)\right) d t \\
& =\mathcal{F}_{p}(f)(\beta)-\mathcal{F}_{p}(f)(\alpha),
\end{aligned}
$$

where (13) holds almost everywhere by Lemma 1. This implies the statement of the proposition.

Corollary 3. Assume $f \in \mathcal{L}^{p}(\mathbb{R})$ and $t f \in \mathcal{L}^{p}(\mathbb{R}) \cap B V_{0}(\mathbb{R})$. Then, by redefining $\mathcal{F}_{p}^{S}(f)$ on a set of measure zero yields

$$
\mathcal{F}_{p}^{S}(f) \in A C G^{*}(\mathbb{R})
$$

Proof. This follows from Proposition 2, [13] [Corollary 1], and [26] [Theorem 2].

Proposition 3. Let $1<p \leq 2$ be fixed, $f \in \mathcal{L}^{p}(\mathbb{R})$ and $t f=h_{p}+h_{0} \in \mathcal{L}^{p}(\mathbb{R})+B V_{0}(\mathbb{R})$. Then, by redefining $\mathcal{F}_{p}(f)(s)$ on a set of measure zero, it yields

$$
\mathcal{F}_{p}(f) \in A C_{\text {loc }}(\mathbb{R} \backslash\{0\}) \cap \mathcal{L}^{q}(\mathbb{R})
$$

and

$$
\frac{d}{d s} \mathcal{F}_{p}(f)(s)=-i\left[\mathcal{F}_{p}\left(h_{p}\right)(s)+\mathcal{F}_{H K}\left(h_{0}\right)(s)\right], \text { a.e. }
$$


Proof. Due to

$$
\frac{1}{\sqrt{2 \pi}} \int_{-n}^{n} e^{-i s t} h_{p}(t) d t \rightarrow \mathcal{F}_{p}\left(h_{p}\right)(n \rightarrow \infty),
$$

there exists $M>0$, such that

$$
\left\|\frac{1}{\sqrt{2 \pi}} \int_{-n}^{n} e^{-i s t} h_{p}(t) d t\right\|_{q} \leq M<\infty
$$

uniformly on $n \in \mathbb{N}$. As argued in Equation (10),

$$
\begin{aligned}
-i \int_{\alpha}^{\beta} \mathcal{F}_{p}\left(h_{p}\right)(s)+\mathcal{F}_{H K}\left(h_{0}\right)(s) d s & =\frac{-i}{\sqrt{2 \pi}} \lim _{k \rightarrow \infty} \int_{-n_{k}}^{n_{k}} \int_{\alpha}^{\beta} e^{-i s t}\left(h_{p}+h_{0}\right)(s) d s d t \\
& =\frac{-i}{\sqrt{2 \pi}} \lim _{k \rightarrow \infty} \int_{-n_{k}}^{n_{k}} \int_{\alpha}^{\beta} e^{-i s t} t f(t) d s d t \\
& =\frac{1}{\sqrt{2 \pi}} \lim _{k \rightarrow \infty} \int_{-n_{k}}^{n_{k}}\left(e^{-i \beta t}-e^{-i \alpha t}\right) f(t) d t \\
& =\mathcal{F}_{p}(f)(\beta)-\mathcal{F}_{p}(f)(\alpha) .
\end{aligned}
$$

where we take a subsequence of $\left(n_{k}\right)$, if necessary. Here, $\alpha, \beta$ are values, such that (12) is valid.

Corollary 4. Assume the hypothesis of Proposition 3. Subsequently, by redefining $\mathcal{F}_{p}^{S}(f)$ on a set of measure zero

$$
\mathcal{F}_{p}^{S}(f) \in A C G_{l o c}^{*}(\mathbb{R})
$$

Proof. Similar arguments, as above, give the result.

Now, we show some examples.

Example 1. Let $\phi(t):=\left(1+t^{2}\right)^{-1}$. It can be verified by a symbolic computation that

$$
\mathcal{F}_{2}(\phi)(s)=\sqrt{\frac{\pi}{2}} e^{-|s|},
$$

and

$$
\frac{1}{\sqrt{2 \pi}} \int_{\mathbb{R}} \frac{t \sin (s t)}{1+t^{2}} d t=-\sqrt{\frac{\pi}{2}} \operatorname{sgn}(s) e^{-|s|} \quad(s \neq 0) .
$$

Applying Proposition 2, we get the equality

$$
\frac{d}{d s} \mathcal{F}_{2}(\phi)(s)=\frac{1}{\sqrt{2 \pi}} \int_{\mathbb{R}} \frac{t \sin (s t)}{1+t^{2}} d t,
$$

which it is directly confirmed, implying the result for the derivative. The integral is in the sense of Henstock-Kurzweil.

Example 2. Let $\psi(t)=\arctan |t|-\frac{\pi}{2}$. Note that $\psi \in B V_{0}(\mathbb{R}) \cap \mathcal{L}^{2}(\mathbb{R}) \backslash \mathcal{L}^{1}(\mathbb{R})$, and $t \psi(t)$ does not belong to $\mathcal{L}^{1}(\mathbb{R})+B V_{0}(\mathbb{R})$. By Theorem 3 , we have that

$$
\mathcal{F}_{2}(\psi)(s)=-\frac{i}{s} \mathcal{F}_{1}\left(\psi^{\prime}\right)(s), \quad(s \neq 0)
$$

Note that $\psi^{\prime} \in \mathcal{L}^{1}(\mathbb{R}) \cap \mathcal{L}^{2}(\mathbb{R})$. Applying Proposition 2 to $\mathcal{F}_{1}\left(\psi^{\prime}\right)$, we get that 


$$
\frac{d}{d s} \mathcal{F}_{1}\left(\psi^{\prime}\right)(s)=-i \mathcal{F}_{H K}(g)(s),
$$

with $g(t)=t \psi^{\prime}(t) \in B V_{0}(\mathbb{R})$. Thus, $\mathcal{F}_{2}(\psi)$ is a continuously differentiable function away from zero and

$$
\frac{d}{d s} \mathcal{F}_{2}(\psi)(s)=\frac{i}{s^{2}} \mathcal{F}_{1}\left(\psi^{\prime}\right)(s)-\frac{1}{s} \mathcal{F}_{H K}(g)(s) .
$$

Example 3. Let $\psi(t):=\sqrt[3]{t} \sin (1 / t)$ and $\varphi(t):=1-C\left(\frac{2}{\pi} \arctan (t)\right)$, where $C$ is the Cantor function [30]. We take

$$
f(t)= \begin{cases}\psi(t) & \text { if } 0<t<1 \\ t^{-1} \cdot \varphi(t) & \text { if } t>2 \\ 0 & \text { otherwise. }\end{cases}
$$

Due to $t^{-1} \cdot \varphi(t)$ belongs to $\mathcal{L}^{p}([2, \infty))$ for $p \geq 1$, it follows that $f \in \mathcal{L}^{p}(\mathbb{R})$, see [31]. Moreover,

$$
\varphi \in B V_{0}([2, \infty)) \backslash \mathcal{L}^{1}([2, \infty)) .
$$

In addition, $g(t)=t \cdot f(t)$ is not in $\mathcal{L}^{1}(\mathbb{R})$, but in $B V_{0}(\mathbb{R})+\mathcal{L}^{1}(\mathbb{R})$. Applying Proposition 2, we have that for $1 \leq p \leq 2$,

$$
\frac{d}{d s} \mathcal{F}_{p}(f)(s)=-i \mathcal{F}_{H K}(g)(s)=-i\left[\mathcal{F}_{H K}\left(g_{1}\right)(s)+\mathcal{F}_{1}\left(g_{2}\right)(s)\right], \quad(s \neq 0),
$$

where $g_{1}(t):=\varphi(t) \cdot \chi_{(2, \infty)}(t)$ and $g_{2}(t):=t \cdot \psi \chi_{(0,1)}(t)$.

\section{Continuity of Operators into $H K(\mathbb{R})$}

Another application of the previous reasoning is to prove the boundedness of some operators.

In [13], it was proved that $\mathcal{F}_{H K}^{C}$ is a bounded operator from $B V_{0}(\mathbb{R})$ into $H K(\mathbb{R})$, whereas $\mathcal{F}_{H K}^{S}$ is not. However, there is a similarity to the result on the boundedness of the operator $\mathcal{F}_{H K}^{C}$, if one considers not exactly $\mathcal{F}_{H K}^{S}$ but a related operator.

Let us consider the Banach space $\mathcal{B}_{1}:=\left\{\varphi \in \mathcal{L}^{2}(\mathbb{R}) \cap A C_{\text {loc }}(\mathbb{R}) \mid \varphi^{\prime} \in B V_{0}(\mathbb{R})\right\}$ with norm defined as

$$
\|\varphi\|_{\mathcal{B}_{1}}=\|\varphi\|_{2}+\left\|\varphi^{\prime}\right\|_{B V} .
$$

Here, $\|\varphi\|_{B V([s, t])}:=\operatorname{Var}(\varphi,[s, t])+|\varphi(u)|$, with $u \in[s, t]$ arbitrarily, but fixed. We recall that the total variation of $\varphi$ on $[s, t]$ is denoted by $\operatorname{Var}(\varphi,[s, t])$ and it is defined as

$$
\operatorname{Var}(\varphi,[s, t]):=\sup \sum_{i=1}^{n}\left|\varphi\left(x_{i}\right)-\varphi\left(x_{i-1}\right)\right|,
$$

where the supremum is taken over all of the partitions $P=\left\{\left[x_{i-1}, x_{i}\right]\right\}_{i=1}^{n}$ of $[s, t]$, see for example [25]. Thus, the norm on $B V(\mathbb{R})$ is defined as

$$
\|\varphi\|_{B V}:=\lim _{s \rightarrow-\infty, t \rightarrow \infty} \operatorname{Var}(\varphi,[s, t])+|\varphi(0)| .
$$

For more details, see $[13,25,32]$.

Theorem 6. The Fourier Sine transform operator $\mathcal{F}_{2}^{S}$ restricted to $\mathcal{B}_{1}$ into $H K(\mathbb{R})$ is a bounded operator. In particular, if $\mathcal{F}_{2}^{S}(f)$ does not change of sign then it is Lebesgue integrable.

Proof. Let $j$ be a nonnegative function with support in $(-1,1)$ and infinitely differentiable, such that 


$$
\int_{-1}^{1} j(t) d t=1
$$

For every $\varphi \in \mathcal{B}_{1}$, we take

$$
\varphi_{\varepsilon}(x):=\int_{-1}^{1} \varphi(x-\varepsilon t) j(t) d t \in \mathcal{B}_{1}
$$

Due to

$$
\varphi_{\varepsilon} \rightarrow \varphi \text { in } \mathcal{L}^{2} \text {-norm, }
$$

it follows that

$$
\mathcal{F}_{2}^{S}(\varphi)(s)=\lim _{\varepsilon \downarrow 0} \mathcal{F}_{2}^{S}\left(\varphi_{\varepsilon}\right)(s) \quad \text { a.e. }
$$

Furthermore,

$$
\frac{d}{d x} \varphi_{\varepsilon}(x)=\int_{-1}^{1} \varphi^{\prime}(x-\varepsilon t) j(t) d t .
$$

It yields, for almost everywhere on $\mathbb{R} \backslash\{0\}$,

$$
\begin{aligned}
\mathcal{F}_{2}^{S}(\varphi)(s) & =\lim _{\varepsilon \downarrow \downarrow} \frac{1}{\sqrt{2 \pi}} \frac{1}{s}\left[\left.\varphi_{\varepsilon}(x) \cos (s x)\right|_{x=-\infty} ^{x=+\infty}-\int_{\mathbb{R}} \cos (s x) \varphi_{\varepsilon}^{\prime}(x) d x\right] \\
& =\lim _{\varepsilon \downarrow 0} \frac{1}{S} \mathcal{F}_{H K}^{C}\left(\varphi_{\varepsilon}^{\prime}\right)(s) \\
& =\frac{1}{S} \mathcal{F}_{H K}^{C}\left(\varphi^{\prime}\right)(s) .
\end{aligned}
$$

Note that, for every bounded interval $[-M, M]$, we have

$$
\varphi_{\varepsilon}^{\prime}(x) \rightarrow \varphi^{\prime}(x) \text { in } \mathcal{L}^{1} \text {-norm }
$$

Additionally,

$$
\operatorname{Var}\left(\varphi_{\varepsilon}^{\prime},|x| \geq M\right) \leq \operatorname{Var}\left(\varphi^{\prime},|x| \geq M-1\right) \rightarrow 0 \text { if } M \rightarrow \infty .
$$

Subsequently, the last equality in (14) follows from continuity of the HK-Fourier Cosine transform in $B V_{0}(\mathbb{R})$. Now, by the Chartier-Dirichlet Test from [14] [Sec. 16, p. 269], we get

$$
\frac{1}{s} \mathcal{F}_{H K}^{C}\left(\varphi^{\prime}\right)(s) \in H K(|s| \geq 1)
$$

Moreover, the Multiplier Theorem implies that

$$
\left\|\frac{1}{s} \mathcal{F}_{H K}^{C}\left(\varphi^{\prime}\right)(s) d s\right\|_{H K(|s| \geq 1)} \leq c_{1}\left\|\mathcal{F}_{H K}^{C}\left(\varphi^{\prime}\right)(s) d s\right\|_{H K(|s| \geq 1)} \leq c_{2}\left\|\varphi^{\prime}\right\|_{B V(\mathbb{R})} .
$$

where $c_{1}, c_{2}$ are positive constants. The last inequality is implied by the continuity of the operator

$$
\mathcal{F}_{H K}^{C}: B V_{0}(\mathbb{R}) \rightarrow H K(\mathbb{R})
$$

Thus, (14) and (15) imply

$$
\left\|\mathcal{F}_{2}^{S}(\varphi)\right\|_{H K(|s| \geq 1)} \leq c_{1}\left\|\mathcal{F}_{H K}^{C}\left(\varphi^{\prime}\right)\right\|_{H K(|s| \geq 1)} \leq c_{2}\left\|\varphi^{\prime}\right\|_{B V(\mathbb{R})}
$$

Since $\mathcal{L}^{2}(\mathbb{R})$ is continuously embedded in $H K_{\text {loc }}(\mathbb{R})$, it yields the result, 


$$
\left\|\mathcal{F}_{2}^{S}(\varphi)\right\|_{H K(\mathbb{R})} \leq c\left(\|\varphi\|_{\mathcal{L}^{2}(\mathbb{R})}+\left\|\varphi^{\prime}\right\|_{B V(\mathbb{R})}\right),
$$

for some positive constant $c$.

We mention that

$$
\mathcal{F}_{2}^{S}\left(W^{1,1}(\mathbb{R})\right)=\mathcal{F}_{H K}^{S}\left(W^{1,1}(\mathbb{R})\right) \not \subset H K(\mathbb{R}),
$$

where $W^{1,1}(\mathbb{R}) \subset B V_{0}(\mathbb{R})$ is the Sobolev space of functions in $\mathcal{L}^{1}(\mathbb{R})$, with derivative in the sense of distributions also in $\mathcal{L}^{1}(\mathbb{R})$. In some sense, Theorem 6 is sharp, see Example 3.12 from [33].

The following corollary follows directly from similar argumentations.

\section{Corollary 5.}

1. The linear operator

$$
\Xi(\varphi)(s):=\frac{1}{s} \mathcal{F}_{H K}^{C}(\varphi)(s)
$$

is a bounded operator from

$$
\mathcal{W}:=\left\{\varphi \in B V_{0}(\mathbb{R}) \mid \Phi^{\prime}(x)=\varphi(x), \Phi \in \mathcal{L}^{2}(\mathbb{R})\right\},
$$

into $H K(\mathbb{R})$.

2. The linear operator

$$
\mathrm{Y}(\varphi):=\frac{1}{S} \mathcal{F}_{1}^{S}(\varphi)(s)
$$

is bounded from $L^{1}(\mathbb{R})$ into $H K(\mathbb{R})$.

Proof. The statement in 1. is a rewording of the previous theorem. Concerning to the claim 2., we note that

$$
Y(\varphi)=-\mathcal{F}_{H K}^{C}(h),
$$

where $h$ is the even extension of the function

$$
\int_{x}^{\infty} \varphi(t) d t, \quad(x>0) .
$$

Subsequently, the result follows from [13].

The previous statements do relate to the results of E. R. Liflyand. He has analyzed Lebesgue integrability of the Fourier transform [5,8,34]. See also [13].

Example 4. If $\varphi \in \mathcal{L}^{1}(\mathbb{R})$ is an odd function, and decreasing or decreasing in $(0, \infty)$ then $\mathrm{Y}(\varphi) \in \mathcal{L}^{1}(\mathbb{R})$. This follows from the previous corollary and the fact that a function in $H K(\mathbb{R})$ that does not change sign it is also in $\mathcal{L}^{1}(\mathbb{R})$, see [35] [Theorem 123]. Furthermore, $\mathcal{F}_{H K}^{C}(h) \in \mathcal{L}^{1}(\mathbb{R})$, where $h$ is the even extension of the indefinite integral of $\varphi$.

\section{Conclusions}

An integral representation of the Fourier transform is obtained on the subspace $\mathcal{L}^{p}(\mathbb{R}) \cap B V_{0}(\mathbb{R}) \cap$ $A C_{\text {loc }}(\mathbb{R}) \backslash \mathcal{L}^{1}(\mathbb{R})$, for $1<p \leq 2$. This is possible by switching to the Henstock-Kurzweil integral. Furthermore, expressions (2), (5) and (6) give explicit formulas of $\mathcal{F}_{p}$ over that subspace. Through our results, specific values of the Fourier transform of particular functions might be approximated with arbitrary accuracy. Moreover, it was shown that $\mathcal{F}_{p}(\phi)(s)$ is differentiable by extending a classical 
theorem in Lebesgue's theory. This illustrates the applicability of the results obtained, which are original in Fourier Analysis over $\mathcal{L}^{p}(\mathbb{R})$. Furthermore, it was proved that the Fourier Sine transform is a bounded operator from a subspace of $\mathcal{L}^{2}(\mathbb{R})$ into the space of Henstock-Kurzweil integrable functions. A related statement has already been proved for the Fourier Cosine transform in [13].

\section{Discussion}

Fourier Analysis is a fundamental theme in Mathematics and applications are everywhere. Generalized integration allows for considering a wider class of functions where Fourier transform operators and other mathematical objects have a sense. The most remarkable about this issue is that it turns out to have implications not only on the new space of functions considered, but also on the classical spaces $\mathcal{L}^{p}(\mathbb{R}), 1 \leq p \leq 2$. In this and other papers, we have shown some implications of these relations. Future research might be to prove similar results for functions of several variables.

Author Contributions: Conceptualization, M.B.; Formal analysis, M.G.M.; Investigation, J.H.A. and M.G.M.; Methodology, J.H.A.; Resources, M.B.; Supervision, M.G.M.; Writing—original draft, M.B. All authors have read and agreed to the published version of the manuscript.

Funding: This research was partially funded by the Czech Science Foundation GA 20-11846S .

Acknowledgments: J.H.A. and M.B. acknowledge partial support from the Mexican Science Foundation CONACYT-SNI.

Conflicts of Interest: The authors declare no conflict of interest.

\section{References}

1. Kurtz, D.S.; Swartz, C.W. Theories of integration. In The integrals of Riemann, Lebesgue, Henstock-Kurzweil, and Mcshane; Series in Real Analysis, 9; World Scientific Publishing Co.: Singapore, 2004.

2. Morales, M.G.; Arredondo, J.H.; Mendoza, F.J. An extension of some properties for the Fourier transform operator on $L^{p}(\mathbb{R})$ spaces. Rev. Un. Mat. Argentina. 2016, 57, 85-94.

3. Bracewell, R. The Fourier Transform and Its Applications, 3rd ed.; McGraw-Hill Book Co.: New York, NY, USA; Toronto, ON, USA; London, UK, 2000.

4. Ruzhansky, M.; Tikhonov, S. Some problems in Fourier analysis and approximation theory. In Methods of Fourier Analysis and Approximation Theory; Ruzhansky, M., Tikhonov, S., Eds.; Birkhäuser/Springer: Cham, Switzerland, 2016; pp. 1-19.

5. Liflyand, E. Functions of Bounded Variation and Their Fourier Transforms. Applied and Numerical Harmonic Analysis; Birkhäuser/Springer: Cham, Switzerland, 2019.

6. Ivashev-Musatov, O.S. On Fourier-Stieltjes coefficients of singular functions. Izv. Akad. Nauk SSSR Ser. Mater. 1956, 20, 179-196.

7. Liflyand, E. Asymptotics of the Fourier sine transform of a function of bounded variation. Math. Notes 2016, 100, 93-99. [CrossRef]

8. Liflyand, E. Integrability spaces for the Fourier transform of a function of bounded variation. J. Math. Anal. Appl. 2016, 436, 1082-1101. [CrossRef]

9. Liflyand, E. The Fourier Transform of a Function of Bounded Variation: Symmetry and Asymmetry. J. Fourier Anal. Appl. 2018, 24, 525-544. [CrossRef]

10. Wiener, N.; Wintner, A. Fourier-Stieltjes transforms and singular infinite convolutions. Am. J. Math. 1938, 60, 513-522. [CrossRef]

11. Kiselman, C.O. Generalized Fourier transformations: the work of Bochner and Carleman viewed in the light of the theories of Schwartz and Sato. In Microlocal Analysis and Complex Fourier Analysis; Kawai, T., Fujita, K., Eds.; World Sci. Publ.: River Edge, NJ, USA, 2002; pp. 166-185.

12. Rudin, W. Real and Complex Analysis; McGraw-Hill: New York, NY, USA, 1984.

13. Arredondo, J.H.; Mendoza, F.J.; Reyes, A. On the norm continuity of the HK-Fourier transform. Electron. Res. Announc. Math. Sci. 2018, 25, 36-47. [CrossRef]

14. Bartle, R.G. A Modern Theory of Integration; Graduate Studies in Mathematics, 32; American Mathematical Society: Providence, RI, USA, 2001. 
15. Monteiro, G.; Slavík, A.; Tvrdý, M. Kurzweil-Stieltjes Integral. Theory and Applications; Series in Real Analysis, Series No. 15; World Scientific Publishing Co.: Singapore, 2019.

16. McLeod, R.M. The Generalized Riemann Integral; Carus Mathematical Monographs, Series No. 20; Mathematical Association of America: Washington, DC, USA, 1980.

17. Grafakos, L. Classical Fourier Analysis, 3rd ed.; Graduate Texts in Mathematics, 249; Springer: New York, NY, USA, 2014.

18. Reed, M.; Simon, B. Methods of Modern Mathematical Physics. II. Fourier Analysis, Self-Adjointness; Academic Press: New York, NY, USA; London, UK, 1975.

19. Debnath, L.; Mikusiński, P. Introduction to Hilbert Spaces with Applications; Academic Press, Inc.: Boston, MA, USA, 1990.

20. Pinsky, M.A. Introduction to Fourier Analysis and Wavelets; Brooks/Cole Series in Advanced Mathematics; Brooks/Cole: Paciffic Grove, CA, USA, 2002.

21. Talvila, E. Henstock-Kurzweil Fourier transforms. Ill. J. Math. 2016, 46, 1207-1226. [CrossRef]

22. Mendoza, F.J.; Morales, M.G.; Escamilla, J.A.; Arredondo, J.H. Several aspects around the Riemann-Lebesgue lemma. J. Adv. Res. Pure Math. 2016, 5, 33-46. [CrossRef]

23. Sánchez-Perales, S.; Mendoza, F.J.; Escamilla, J.A. Henstock-Kurzweil integral transforms. Int. J. Math. Math. Sci. 2012, 2012, 209462. [CrossRef]

24. Gordon, R.A. The Integrals of Lebesgue, Denjoy, Perron, and Henstock; Graduate Studies in Mathematics, 4; American Mathematical Society: Providence, RI, USA, 1994.

25. Leoni, G. A First Course in Sobolev Spaces; Graduate Studies in Mathematics, 105; American Mathematical Society: Providence, RI, USA, 2009.

26. Talvila, E. Necessary and sufficient conditions for differentiating under the integral sign. Am. Math. Mon. 2001, 108, 544-548. [CrossRef]

27. Ashordia, M. On the Opial type criterion for the well-posedness of the Cauchy problem for linear systems of generalized ordinary differential equations. Math. Bohem. 2016, 141, 183-215. [CrossRef]

28. Groh, J.A. Nonlinear Volterra-Stieltjes integral equation a Gronwall inequality in one dimension. Ill. J. Math. 1980, 24, 244-263. [CrossRef]

29. Gasquet, C.; Witomski, P. Filters and Transfer Functions. In Fourier Analysis and Applications. Filtering, Numerical Computation, Wavelets; Springer: New York, NY, USA, 1999; pp. 11-19.

30. Corothers, N.L. Real Analysis; Cambridge University Press: Cambridge, UK, 2000.

31. Gordon, R.A. Some integrals involving the Cantor function. Am. Math. Mon. 2009, 116, 218-227. [CrossRef]

32. Mendoza, F.J. On pointwise inversion of the Fourier transform of $B V_{0}$ functions. Ann. Funct. Anal. 2010, 1, 112-120. [CrossRef]

33. Arredondo, J.H.; Reyes, A. Interpolation Theory for the HK-Fourier Transform. Rev. Un. Mat. Argentina 2020, accepted for publication.

34. Liflyand, E.R. On asymptotics of Fourier transform for functions of certain classes. Anal. Math. 1993, 19, 151-168. [CrossRef]

35. Titchmarsh, E.C. Introduction to the Theory of Fourier Integrals; Clarendon Press: Oxford, UK, 1937.

(c) 2020 by the authors. Licensee MDPI, Basel, Switzerland. This article is an open access article distributed under the terms and conditions of the Creative Commons Attribution (CC BY) license (http://creativecommons.org/licenses/by/4.0/). 